\title{
The Theory of Processes Optimal CONTROL AND OPERATOR EQUATIONS
}

\section{JADlOVsKa, A.; KATALINIC, B.; Hrubina, K. \& WeSSELY, E.}

Abstract: The chapter presents the results of the original and applicative character obtained by the authors in mathematical theory of processes optimal control with the use of informatics. The authors have applied a modern approach to informatics while investigating the application of information technology to processes control. The chapter deals with the theory of systems, continuous and discrete tasks of dynamic optimization, modeling and models classification, mathematical models of complex systems. Via operator equations, the task of the optimal control of systems with distributed parameters has been defined. The designed algorithm has been applied to the solution of the problem of optimal control of a body heating process. In addition, there is included a brief characteristic of the algorithms designed by the authors based on different optimization methods for the numerical solution of the defined problems of processes optimal control with the use of $P C$.

Key words:, modeling, mathematical model, optimal control problem, operators equations, algorithms
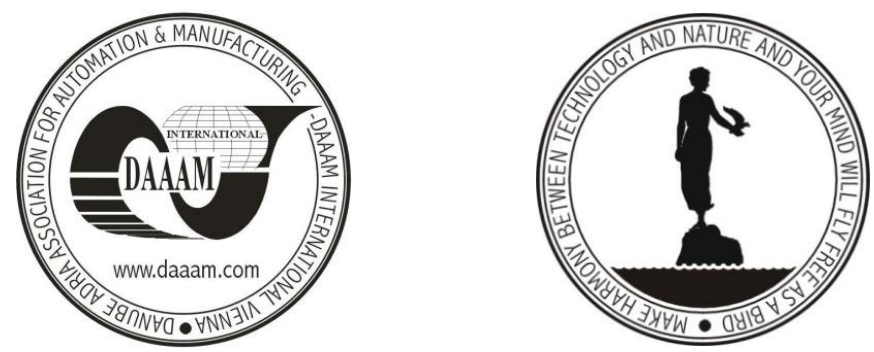

Authors' data: Assoc. Prof. PhD. Jadlovska, A[nna]*, Univ. Prof. Dipl.-Ing. Dr.h.c.mult. Dr.techn. Katalinic, B[ranko]**, Assoc. Prof. PhD. Hrubina, $\mathrm{K}[\mathrm{amil}] * * * ;$ Assoc. Prof. CSc. Wessely, E[mil]****,*Technical University of Kosice, Letna 1, Kosice, Slovakia,** University of Technology, Karlsplatz 13, 1040, Vienna, Austria, ***Informatech Ltd., Košice, Slovakia, ****University of Security Management in Kosice, Slovakia, anna.jadlovska@tuke.sk, katalinic@mail.ift.tuwien.ac.at, kamil.hrubina@tuke.sk, emil.wessely@vsbm.sk

This Publication has to be referred as: Jadlovska, $\mathrm{A}[\mathrm{nna}]$; Katalinic, $\mathrm{B}[$ ranko]; Hrubina, K[amil] \& Wessely, E[mil] (2014). The Theory of Processes Optimal Control and Operator Equations, Chapter 03 in DAAAM International Scientific Book 2014, pp.033-050, B. Katalinic (Ed.), Published by DAAAM International, ISBN 978-3-901509-98-8, ISSN 1726-9687, Vienna, Austria

DOI: $10.2507 /$ daaam.scibook.2014.03 


\section{Introduction}

In recent years, in connection with the development of computing and information technology, there has been an increasing emphasis on designing qualitatively new control systems, namely optimal control systems, according to the selected quality indicator, eventually multiple indicators. This fact implies the development of the optimal control theory of processes, systems. In this scientific area, the original works were written by Athans, Bellman, Butkovskiy, Lions, Mesarovic, Pontryagin and others. The scientific bases of the control theory were created by an American mathematician Norbert Wiener and published in his book. N. Wiener is the founder of cybernetics, whose object is the investigation of control and information processing in technical systems, living organisms and collectives composed of living organisms and technical systems. Another well-known definition of cybernetics is by A.N. Kolmogorov, who defines cybernetics as a science dealing with the study of the systems of an arbitrary nature that are able to receive, store, process information and use it for the control and regulation. Concurrently with the development of cybernetics, the basics of information theory were created (C.E. Shannon), which gradually led to the origin of a new field - informatics.

In this chapter, we will apply the definition of informatics, which reflects a modern approach to informatics. Its author is the American Society for Information Science: "Informatics deals with the formation, gathering, organization, interpretation, storing, searching, extension, manipulation and use of information with a particular regard to the application of modern technology in these areas. It has both purely scientific (theoretical) components, which investigate an object regardless of the application, and application (practical) components which contribute to the development of services and products." It should also be realized that nowadays the process of informatization (i.e. the introducing of informatics to a social practice) is one of the characteristics of the developed countries in the world. Informatization is now a global process associated with the principal changes in the structure and character of a global, economic and social development as well as the transition to the new forms of information exchange. This process involves in varying degrees the global community, it affects most of the activities spheres and substantially changes the character of its development, economic relations, standard and quality of living of the society. Thus, informatization is the process of information and information technology penetration and utilisation in all areas of a social life with the aim of achieving its effective functioning. It is exhibited through a complex, overall and crucial effect of informatics on the economy, industry, state and local administration, culture, education, transport, market, scientific and technological development, health service, consumption and standard of living and in general on the whole of society and all of its spheres in the given state or a group of states.

The aim of the chapter is to apply a modern definition of informatics and investigate the application of information technology to the processes control while dealing with the systems theory and the designing of algorithms for their optimal control. Another objective is to create a stable algorithm based on the latest results of 
the mathematical control theory for solving the defined problems of the optimal control of distributed parameter systems. To present the designed algorithm with the application to the solution of the problem of optimal control of the body heating process. Furthermore, to describe briefly the algorithms designed by the authors based on various optimization methods for the numerical solution of the defined problems of processes optimal control with the use of PC.

\section{Generally on information technology and optimization of processes control}

Nowadays, in a new economic environment, enterprises and private firms must constantly solve the problem of productivity and profitability. There are special demands made of enterprises and firms arising from a continuous technological development. In addition, the conditions of firms and enterprises are getting worse due to rapid changes on the sales markets as well as the growing requirements of customers on the quality of products offered for sale. For these reasons, the competitiveness and economic development of the firm or enterprise, become to a substantial degree dependent on information and control systems of an enterprise or a firm. It is necessary to realize that the information and control system is a set of personnel, procedures and resources that gathers, transforms and distributes information, eventually the means provided for the organization control so as to achieve the goals laid out. Such a system, of course, is created and developed with a significant support of information technology techniques and methods. It means that within these contexts we highlight a principal linking of information and information flows with the processes ensuring the control and development of the enterprise or a firm in accordance with the determined actual strategic objectives. Basically, information technology allows and promotes the effective use of mathematical methods, operational analysis, optimal programming and optimization methods in the control of production firms, enterprises, or firms providing services. A scientific discipline dealing with the analysis of processes related to the control, functioning and designing of complex socio-economic-technical systems, i.e.organizational units, in which people and technical equipment are interconnected and in which there are economic, social, technical and material linkages, is called operational research.

An example of such a system is an industrial plant, a public transport system, a system of health service, etc. Operational research is significant in the state administration control, in solving relationships between the economic growth and the environmental quality. The availability of operational research methods has been growing especially in recent decades along with the intensive introduction of personal computers that is connected with the creation of the systems to support the managerial decision making, e.g. (Hrubina \& Taufer,2005). The rising importance of their utilisation is manifested in the operational and strategic planning, in all phases of project control, manufacturing processes control, a prognostication as well as in organizational structures and information systems designing. In operational research, the mathematical techniques are used to model the tasks that arise under the systems control. For this purpose, a system approach and a team of specialists are used. 


\section{A system, static and dynamic systems and optimization tasks}

Within the theory of dynamic optimization, the following considerations will the derived from the concept of the system. The system will be called a structure $(S, R)$, where $S$ is the set of elements, and $R$ is the set of relations between them. In addition to the Mesarovic's definition, let us give an equivalent definition: The system is a propositional formula $S(x)$ producing true statements $V_{1}(x), V_{2}(x), \ldots, V_{m}(x)$ about the mutual relations of elements and a system environment, including a system state. Based on the definition it follows that according to the nature of these propositions formalization, the systems can be categorized. The given definition of the system is also of a great practical importance especially for our considerations, since in many systems, only formalized prepositions, such as functions, differential equations, integral equations, etc. are examined.

Thus, the systems can be classified according to various aspects. Depending on whether the system state changes over time or it does not, we distinguish between dynamic systems and static systems. A dynamic system, whose state change can be influenced, is called controllable. Otherwise, the system is non-controllable. Furthermore, we will deal with dynamic and controllable systems. With mathematical models, we cannot work directly with the "state" of the system, but only with a certain information about its state. We therefore will assume that the system state at any instant of time may be described by the n-tuplet of the arranged real numbers, which is called the state vector:

$$
\boldsymbol{x}^{T}(t)=\left(x_{1}(t), x_{2}(t), \ldots, x_{n}(t)\right)
$$

The set of all permissible states, which is determined by the conditions of a particular task, is called a state space. The controllability of a dynamic system can be described as follows: each particular function $\boldsymbol{u}(t)$ of the set $\boldsymbol{U} \subset \boldsymbol{R}_{m}$, according to the prescribed rule, can be unambiguously assigned the state vector $\boldsymbol{x}(t+\Delta t)$. This means that the selection of the function $\boldsymbol{u}(t)$ at time $\mathrm{t}$ affects the state of the system at time $(t+\Delta t)$. The function $\boldsymbol{u}(t)$ with the given property will be called a control (sometimes also

such names as politics, strategy are used). The set of which the controls can be chosen will be called the controls space. In fact, we may consider two types of dynamic optimization tasks.

\subsection{A continuous task of a dynamic optimization}

Let a real object (a system) be given and let the sets $\boldsymbol{X}, \boldsymbol{U}, \boldsymbol{P}, \boldsymbol{C}$ be given, where $\boldsymbol{X}$ is the state space, $\boldsymbol{U}$ is the control space, $\boldsymbol{P}$ is the set of the initial states and $\boldsymbol{C}$ is the target set. Let the state of an object (a system) be described by the state vector $\boldsymbol{x}(t) \in \boldsymbol{X}, \boldsymbol{X} \subset \boldsymbol{R}_{n}$ and the input control vector of the real object $\boldsymbol{u}(t) \in \boldsymbol{U}, \boldsymbol{U} \subset \boldsymbol{R}_{n}$. The behaviour of the object (a dynamic process) is described by a set of differential equations

$$
\dot{x}_{i}(t)=f_{i}(\boldsymbol{x}(t), \boldsymbol{u}(t), t), \quad i=1,2, \ldots, n
$$


The basic task of the dynamic optimization is to determine of all the controls $\boldsymbol{u}(t) \in \boldsymbol{U}$ in the interval $\left\langle 0, t_{f}\right\rangle$ and of all the solutions $\boldsymbol{x}(t) \in \boldsymbol{X}$ on $\left\langle 0 . t_{f}\right\rangle$ of the equations (1), with the initial state of the given set $\mathrm{P}$ and the final state of the given target set $\mathrm{C}$,

$$
\begin{aligned}
& x(0) \in P, \quad x(0)=x_{0} \\
& x(t) \in C, \quad x\left(t_{f}\right)=x_{f}
\end{aligned}
$$

such control $\boldsymbol{u}(t)$ for which the criterial (objective) function

$$
J(\boldsymbol{x}(t), \boldsymbol{u}(t))=\int_{0}^{t_{f}} f_{0}(\boldsymbol{x}(t), \boldsymbol{u}(t), t) d t
$$

takes a minimum value. Such control $\boldsymbol{u}(t)$ will be called optimal. The function $f_{0}(\boldsymbol{x}(t), \boldsymbol{u}(t), t)$ and the functions $f_{i}(\boldsymbol{x}(t), \boldsymbol{u}(t), t)$ are given and generally are non-linear. Based on the mathematical expression (3), it is clear that the criterion of efficiency, or a criterial (objective) function, in addition to the function in a "classical" meaning, can also be the representation where the set of arguments is the set of functions. Then we speak of thefunctional, (Tripathi, 2008),(Jadlovská,A. \& Jadlovská,S., 2013).

\subsection{Discrete task of a dynamic optimization}

A discrete task of a dynamic optimization can be formulated similarly to a continuous task, but the difference is that the sets of differential equations (1) are expressed in a discrete form and likewise the criterial (objective) function (3). To formulate a discrete task of a dynamic optimization, let us assume the given object (a system) and the given sets X,U,P and C. The behaviour of the object (a dynamic process) is described by the equations in the form

$$
x_{i+1}=F_{i}\left(x_{i}, u_{i}\right), \quad i=0,1, \ldots, k-1
$$

The aim of a discrete task solving is to find among all the controls $\left\{\boldsymbol{u}_{i}\right\}$ and all the solutions $\left\{\boldsymbol{x}_{i}\right\},(i=0, \ldots, k)$ of the equations (1a) with the initial state of the given initial set $P$ and final state of the given target set $C$

$$
x_{0} \in P, \quad x_{k} \in C
$$

which comply with the state constraints

$$
x_{i} \in X_{i}, \quad i=1, \ldots, k-1
$$

such control $\left\{\boldsymbol{u}_{i}\right\}$ for which the criterial (objective) function

$$
J\left(\left\{x_{i}\right\},\left\{u_{i}\right\}, k\right)=\sum_{i=0}^{k-1} f_{O i}\left(x_{i}, u_{i}\right)
$$


takes a minimum value. Such control will be called optimal. It is obvious that the functions $f_{0, i}\left(x_{i}, u_{i}\right), F_{i}\left(x_{i}, u_{i}\right)$ are known. Continuous and discrete tasks of a dynamic optimization can be divided into tasks with a fixed time horizon ( $t_{f}$ is fixed) and the tasks with a free time horizon ( $t_{f}$ is not fixed). The definitions of dynamic optimization tasks that we have mentioned are so general that the variation tasks, the control theory tasks and dynamic programming tasks represent their individual cases. There is a variety of methods to solve the problems of optimal control and the most important are:

1) Calculus of variations ,(Athans

$\&$

Falb,1966),

2) Dynamic programming (Bellman's principle of optimality), (Bellman, 1967)

3) Pontryagin's minimum (maximum) principle, (Pontrjagin et al. 1983)

4) The method for aggregating state variables

5) The methods of a functional analysis, etc., (Poznyak, 2008), (Jadlovská,A. \& Jadlovská,S., 2013).

\section{Modeling and models classification}

Models generally represent a wide and considerably varied area of means and ideas that differ in terms of their function as well as the method of their implementation. A model is a formal or a materialized entity that represents some aspects of the original (a model system, a modelled system) and that is purposefully designed to be used in the study and the deliberate influencing of the modelled system. Modeling is then a process, when to the examined system, i.e. an object, original, is according to certain criteria assigned other system, which is called a model. Model is an isomorphic or homomorphic representation of an object in a selected objective set transferring object characteristics adequately to the representation, (Jadlovská, et al., 2011).

\subsection{Classification of models}

The issue of modeling is very wide and therefore we will focus only on some classification aspects:

a) Classification of models according to the degree of abstraction: According to the degree of abstraction from a real object, all object models can be divided into several groups. In terms of maintaining the original process, we distinguish substance, structural and functional models. Substance models, including natural models, physical models and physical analog, maintain the original process in the substance. With structural models, it is replaced by the structure and with functional (cybernetic) models by the functionals of external behaviour. Each of the mentioned models can be analog, digital or hybrid, eventually, considering a different aspect, deterministic or stochastic.

a1) Natural models (zero degree of abstraction). The physical process is entirely maintained in the substance, including the carrier substance. When modelling, all variables dimensions are considered unitary. Basically, it is the original, the object under test conditions corresponding to the studied operating conditions. These models 
can only be complete. An example may be a steel slab unevenly heated along the length. The detection of the temperature $\mathrm{T}$ at any part on the slab can be carried out by a direct measurement.

a2) Physical models (1st degree of abstraction). The physical process is maintained wholly or partly in the substance, the carrier may be different. The determinative are the laws of a physical similarity. The dimensions of variables in modelling are not unitary. The mathematical description is identical to the mathematical description of the original, but it is not required for the solution. These models may be complete, incomplete or approximate. An example may be a physical model of a slab replaced by a physically similar body. The heat conduction corresponds to the heat conduction. The physical similarity includes geometric similarity, the similarity of physical properties and that of boundary and initial conditions. Temperatures on the original $(\mathrm{T}, \mathrm{T} 1, \mathrm{~T} 2)$ correspond to the temperatures on the model (TM, T1M,T2M).

a3) Physical analog (2nd degree of abstraction). The original physical process is replaced by an analogous process. The substance of the process is maintained in analogy. The laws of a mathematical similarity (isomorphism in equations) are determinative. The laws of the physical similarity apply in whole or partly. Basic equations are solved in the original or a differential form. From the viewpoint of space and time, they may be continuous, discrete, or combined. An example may be the process of heat conduction in a slab which is replaced for example by electrical current conduction by a conductor, i.e. by a physical- analogous process which is based on a mathematical similarity of both basic equations. Slab temperatures (T, T1, $\mathrm{T} 2)$ correspond to the electric voltage (U, U1, U2) of the models.

a4) Structural model (3rd degree of abstraction). The substance of the original physical process is replaced by the structure. The mathematical similarity of the structures, i.e. of input and output, is determinative. Basic differential equations are usually converted to a set of ordinary differential or algebraic equations, possibly corresponding difference equations. Example: a structural analog of the slab is represented by an analog, digital or hybrid model, eventually by a computer where the process of heat conduction is carried out in the structure. This means that the similarity is maintained only at certain $(1,2, \ldots, n-1, n)$, corresponding to respective parts on the slab.

a5) Functional models (a higher degree of abstraction). The process is considered neither in the substance, nor in the structure, or in physical and mathematical similarity. An object is considered a black box. The similarity in the external behaviour of the system, i.e. the similarity of the functionals, is determinative. Essentially, the function of the function is modelled. Functional (cybernetic) models may comprise of one or more elements (sets) or may be combined. An example may be a single-element single-system functional model of the heated slab, which is represented by a black box, to the input of which a function of temperature $f(\mathrm{~T} 1)$ is supplied and at the output we obtain $f(T 2)$. In fact, the function $F[f(T 1)]$ is modelled which depends for example on the dimensions of the slab, physical properties of the material, external conditions, and the like. With a two- 
element functional model of the heated slab it refers to the expression of the description of the function of the temperature TA effect of the slab A on another system B, which may be another slab, or some other body, a person, etc. Similarly, there exist multi-element models or functional models Multiset of the heated slabs sets. This case refers to the expression of the function of the effect of the resultant temperature TMA of the slabs set MA on a set MB, or on other objects.

b) The classification of models according to their use in a control process:

b1) Functional models simulate the behaviour of the modelled system under certain defined effects of the environment. Functional models to a large extent are used for simulated dynamical systems.

b2) Directive models are formed independently of a modelled dynamic system and serve as instructions under which the processes in the original run.

b3) Descriptive models are directly derived from the original dynamic system and express the conditions, state or behaviour of the original system.

b4) Structural models divide a complex system (a complex) into the appropriate subsystems between which there are characteristic relations. Subsystems may also represent complex systems which allow further structural modeling. Thus, hierarchical models of a complex system are formed that in the best way describe the properties and the effect of a complex system because they are the reflection of one of the essential features of complex systems.

c) The classification of models according to time relations:

c1) Prognostic models (extrapolation models), which show the predicted future development of the modelled system. They can be divided into:

- factographic, which serve only for processes extrapolation running in a modelled system,

- planning, which are patterns according to which a structure or a dynamics of the original, i.e. of the modelled system, are created.

c2) Actual models operate in real time, i.e. they are "concurrent" with the processes in the original to such extent that they can be used for influencing the work of the modelled system.

c3) Retrospective models represent a previous condition or previous dynamic processes of the modelled system. For the use of models in the control processes, the relationship of the original model and its model is significant, particularly from the standpoint of accuracy and reliability of the model. In the control and monitoring of technological and other special-purpose processes, a large amount of models is used. Let us give more examples: The scheme of a technological process is a directive prognostic model of the technological process. The process of data gathering in the technological system provides a descriptive actual model of the dynamic process ongoing in the process. The technical specification, performance and manufacturing characteristics, the structure and disposition of the plant constitute the functional model, which is the basis for the creation of a prognostic model of the manufacturing process, (Jadlovská \& Hrubina, 2011). 


\section{Mathematical models of complex systems}

Mathematical models of complex systems, complexes, due to the mentioned classification, may be divided into two classes:

a) deterministic models

b) stochastic models.

Mathematical models of complexes can also be classified according to the continuity or discontinuity :

a) discrete space-time models, eventually continuous - time models

b) continuous - space models and discrete-time models eventually continuous time models.

According to the abstraction, which is used in the complexes description, we distinguish the following types of complex systems descriptions:

Symbolic, i.e. linguistic description

Description from the standpoint of the theory of sets. In this description, a general system is the complex which is considered a certain subset of the Cartesian product of sets.

Abstract algebraic description. This case refers to the application of mathematical apparatus of abstract algebra and topology.

A description from the standpoint of information theory. The description is especially aimed at the flows of information in the complex.

A description from the standpoint of mathematical logic. This refers to the use of algorithms for the complexes description. This description is motivated by the use of control computers to control complexes.

A description from a dynamic aspect of the control theory. In this case, a complex is considered a dynamic system and its description is based on the automatic control theory (e.g. the theory of multidimensional linear systems, stability theory, the theory of systems with distributed parameters) or some methods of operational research (e.g. dynamic programming).

A heuristic description. This description is used especially where a part of complexes is a person or more people and it is necessary to express or model their behaviour and their activities. Furthermore, from the viewpoint of a mathematical description, complex systems (complexes) can be divided into dynamic systems in a state space and sequential machines.

Currently, in the theory of dynamic systems, the sets of differential equations are used to describe the systems which are not particularly suitable for the complex systems description. Therefore, for the description of systems with a large number of variables, new methods are searched for, e.g. a method based on the regression analysis and a polynomial theory of dynamic systems, (Hrubina, \& Jadlovská, 2004). 


\subsection{Physical models of complex systems}

It is possible to create models of complex systems which are based on the analogy to some events in the complexes and some other physical phenomena. The analogy consists in having the same mathematical description. Such physical interpretation of the mathematical description of events in complex systems is called a physical model. Physical models are important because they allow a more general view of the relations between events in various systems.

The best-known physical models of complexes dynamics are:

- flow models

- diffusion models.

\subsection{A mathematical model and a real process}

A mathematical model of a real process designed from the cybernetic aspect has to provide the information in particular about:

- the relation between input and corresponding output signals and

- the structural arrangement of the individual members of the system and the character of their mutual bonds.

From the viewpoint of a mathematical representation of the control problems, we distinguish two types of models descriptions:

a) external model of the process

b) internal model of the process.

For the external model, the so-called macro approach is used, otherwise expressed, this model is based on input-output relations and does not immediately reflect the internal structure of the systems with internal variables that are called state variables (or variables). This approach is very simple and clear. For the internal model, the micro approach is used, which is expressed by the relations input - state output. This approach is more ferquently utilised with a computer-based control.

\section{On problems solution of the systems with distributed parameters control}

We know that each task of an optimal control of the distributed parameters system is basically a task of a non-classical variational calculus, (Hulko et al. 1998).

Throughout this chapter we use the notational conventions for spaces , operators etc. adopted by (Lions, 1986).

\subsection{The control of systems described by parabolic partial differential equations}

Let $V$ and $H$ be the two Hilbert's spaces on $R$ in which the norms \|\|$_{V}$ and \|\|$_{H}$ and the corresponding dot products $((., .))_{V}$ and $((., .))_{H}$ are indicated. Let $V \subset H$, grouted representation from $V$ to $H$ be continuous; and $V$ be dense in $H$, i.e. there exists such a constant $c$ that

$$
|v| \leq c\|v\|
$$

The space $H$ is identified with its dual space. Let indicate by the help of $V^{\prime}$ a dual space to $V$. Then it is possible to identify $H$ as a subspace of $V^{\prime}$ :

$$
V \subset H \subset V^{\prime}
$$


whereas each space is dense in the following one having a continuous representation.

Consider further a Hilbert space $U$ which we shall call the control space and the dual space $U^{\prime}$. The variable $t$ will be used to represent time. We shall assume that $t \in\left(0, t_{f}\right)$ where $t_{f}<\infty$. Given a set of bilinear forms continuous on $V: \varphi, \psi \rightarrow a(t ; \varphi, \psi) ; t \in\left(0, t_{f}\right)$. With respect to this set we assume that for every $\varphi, \psi \in V$ the function $t \rightarrow a(t ; \varphi, \psi)$ is measurable on $\left(0, t_{f}\right)$ and that

$$
|a(t ; \varphi, \psi)| \leq C\|\varphi\| .\|\psi\|
$$

There exists a number $\lambda$ such that

$$
a(t ; \varphi, \psi)+\lambda|\varphi|^{2} \geq \alpha\|\varphi\|^{2}, \alpha>0 ; \forall \varphi \in V, t \in\left(0, t_{f}\right)
$$

For every $t$ we may write

$$
a(t ; \varphi, \psi)=(A(t) \varphi, \psi), \quad A(t) \varphi \in V^{\prime}
$$

where (...) is the scalar products of elements belonging to $V^{\prime}$ and $V$.

Let $L_{2}\left(0, t_{f} ; V\right)$ be the space of measurable functions $t \rightarrow f(t)$ mapping the interval $\left(0, t_{f}\right)$ into $V$ such that

$$
\left(\int_{0}^{t_{f}}\|f(t)\|^{2} d t\right)^{\frac{1}{2}}<\infty
$$

Analogously we introduce the notion of the space $L_{2}\left(0, t_{f} ; V^{\prime}\right)$. Let the operator $A(t) \in \mathscr{L}\left(L_{2}\left(0, t_{f} ; V\right) ; L_{2}\left(0, t_{f} ; V^{\prime}\right)\right)$ and $A^{*}(t)$ the associated operator.

Further let $\Lambda$ and $\Lambda_{U}$ be the canonical isomorphism from $H$ onto $H^{\prime}$ and from $U$ onto $U^{\prime}$.

$$
\begin{aligned}
& C \in \mathscr{L}\left(L_{2}\left(0, t_{f} ; V\right) ; H\right) ; \quad B \in \mathscr{L}\left(U ; L_{2}\left(0, t_{f} ; V^{\prime}\right)\right) \\
& N \in \mathscr{L}\left(U, U^{\prime}\right) ; \quad(N u, u)_{U} \geq v\|u\|_{U}^{2}
\end{aligned}
$$

where $(.,$.$) , is the scalar product of U$ and $U^{\prime}$. Further let the criterial function have the form

$$
J(v)=\left\|C y(v)-z_{d}\right\|_{H}^{2}+(N v, v)
$$

where $z_{d}$ is a given element of $H$. The set of feasible controls is $U_{p}$ convex and closed in $U$. In this case as well as previously the optimal control problem consists in finding a control $u \in U_{p}$ which minimizes the functional $J(v)$, i.e.

$$
J(u)=\underset{v \in U_{p}}{\operatorname{in}} J(v)
$$

Theorem 1. Assuming (3), (4) and (6) are satisfied and $C \in \mathscr{L}\left(L_{2}\left(0, t_{f} ; V\right) ; H\right)$; the optimum control $u, u \in U_{p}$, is characterized by the equations

$$
\begin{aligned}
\dot{y}(t, u)+A(t) y(t, u) & =f(t)+B u(t), \quad y(0 ; u)=y_{0} \\
-\dot{\psi}(t, u)+A^{*}(t) \psi(t, u) & =C^{*} \Lambda\left(C y(t, u)-z_{d}\right) ; \quad \psi\left(t_{f}, u\right)=0
\end{aligned}
$$


Jadlovska, A.; Katalinic, B.; Hrubina, K. \& Wessely, E.: The Theory of Processes ...

$$
\left(\Lambda^{-1} B^{*} \psi(t, u)+N u, v-u\right)_{U} \geq 0, \quad \forall u, v \in U_{p}
$$

where $y(t, u) \in L_{2}\left(0, t_{f} ; V\right) ; \quad \psi(t, u) \in L_{2}\left(0, t_{f} ; V\right)$,

(Lions, 1968).

6.2 Optimal control of processes with distributed parameters and the problem formulation

Every optimum system control problem is in general expressed through a mathematical model of the process or of the system under control and through a criterial functional. In the sequel we shall consider a mathematical model of a process described by a bidimensional partial defferential heat conduction equation

$$
\text { ( } . c) \frac{\partial \varphi(M, t)}{\partial t}=\left(C^{i j} \Delta \varphi(M, t)\right)+f(M, t)
$$

where $M$ is a point with coordinates $\left(x_{1}, x_{2}\right)$ in a bounded region $\Omega \subset R^{2}$ (Euclidean $\quad$ space), and $t$ denotes time. The function $\varphi(M, t) \in\left(\Omega \times\left\langle 0, t_{f}\right\rangle\right)$ represents the distribution of temperature as a function of coordinates $\left(x_{1}, x_{2}\right)$ and time t. $C^{i j}$ are the elements of the heat conductivity matrix (the contravariant components of symmetrical tensor of order two, $C^{i j}=C^{j i}$, which is assumed to be positive definite). ( $\rho . c)$ is the heat capacity, $\rho$ is the specific mass, $c$ the specific heat, $f(M, t)$ is a function for distribution control. The initial conditions $\varphi(M, 0)=\varphi_{0}(M)$ specifies the initial distribution of temperature in $\Omega$ at the time $t=0$.

We may consider, broadly speaking, several kinds of boundary conditions: basic, time-varying, non-linear and combined. The boundary conditions are considered according to the nature of the problem being solved: Dirichlet, Neumann, Fourier and mixed ones. Let us consider boundary conditions taking the form:

$$
\begin{gathered}
\alpha \varphi(0, t)+(1-\alpha) \varphi^{\prime}(0, t)=g_{1}(t) \quad \text { for } \quad M \in \Omega \\
\beta \varphi(1, t)-(1-\beta) \varphi^{\prime}(1, t)=g_{2}(t)
\end{gathered}
$$

where $\alpha, \beta$ are the constants and $g_{1}(t), g_{2}(t)$ are the given functions of the variable $t$. The initial conditions are expressed in the form:

$$
\varphi(M, 0)=\varphi_{0}(M) \quad \text { for } \quad M \in \bar{\Omega}
$$

where $\varphi_{0}(M)$ is the given function.

For an optimal process control of heat conduction we consider the functional defined in the form of the integral criterion:

$$
J(\varphi, u)=\int_{0}^{t_{f}}\left\{\|\varphi(t)\|_{L_{2}(\Omega)}^{2}+\|u(t)\|_{L_{2}(\bar{\Omega})}^{2}\right\} d t+\gamma\left\|\varphi\left(t_{f}\right)\right\|_{L_{2}(\Omega)}^{2}
$$

where $\gamma=0$ if $t_{f}=\infty, \quad \gamma=1$ if $t_{f}<\infty$. 
With the problems of optimal control of distributed parameter processes, which are defined by the relations (9)-(12), the control function $u(t)$ may act directly or indirectly in the subintegral function of a minimum functional (through the boundary conditions), (Lasiecka \& Triggiani, 2000), Jadlovská \& Hrubina, 2011), (Jadlovská,A. \& Jadlovská,S., 2013).

If we consider the material heating simulation techniques then the mathematical model of the process we are describing has to take into account heat transfer by emission which is expressed by the fallowing nonlinear boundary condition:

$$
\begin{gathered}
\lambda \varphi^{\prime}(S, t)=\sigma_{1}\left\{\left[u_{1}(t)\right]^{4}-[\varphi(S, t)]^{4}\right\}+\alpha_{1}\left[u_{1}(t)-\varphi(S, t)\right] \\
-\lambda \varphi^{\prime}(-S, t)=\sigma_{2}\left\{\left[u_{2}(t)\right]^{4}-[\varphi(S, t)]^{4}\right\}+\alpha_{2}\left[u_{2}(t)-\varphi(S, t)\right]
\end{gathered}
$$

where:

$\lambda$ is the heat conductivity of the material,

$\sigma$ the coefficient of heat transfer by emission

$\alpha$ the coefficient of heat transfer by convection

$u(t)$ the medium temperature in the furnace;

The subscripts 1 and 2 denote the upper or lowe surface of the heated material, respectively.

Furthermore, for purposes of simulation for minimization of losses due to oxidation during heating we may define an integral criterion as follows:

$$
\begin{aligned}
& J \equiv p^{2}=\int_{0}^{t_{f}} \alpha_{s} R\left[\varphi(S, t)-H_{\varphi}\right]^{2} d t \\
& R=1 \quad \text { if } \quad \varphi(S, t) \geq H_{\varphi} ; R=0 \quad \text { if } \quad \varphi(S, t)<H_{\varphi}
\end{aligned}
$$

$p$ is the surfase overburn of the metal, $\varphi(S, t)$ the surfase temperature of the metal, $H_{\varphi}$ the limit temperature for scale formation, $\alpha_{s}$ the aggressivity coefficient of combustion product ( $H_{\varphi}$ and $\alpha_{s}$ are considered as given for the purposes of simulation).

In this case the problem is to propose an algorithm for solving the mathematical

model expressed by (9), the given initial and boundary conditions according to the problem type which may be considered e.g. as given by (13) and the selected optimality criterion in the integral form (14). The result of the solution in a given time interval will be the time dependent control function $u(t)$ (the medium temperature inside the furnace), as well as the time dependences of the temperature of surfaces and center of the heated material, (Jadlovská et al. 2012; Hrubina , 2003).

It is necessary to transform the described mathematical model to a classical linear dynamic state model in order to desing optimal control of the investigated process with distributed parameters. The solution to such a model allows to obtain the programme optimal control $u^{*}(t)$ and optimal feedback control $u^{*}(t, x(t))$ as well as the optimal trajectory of the controlled process. 
Jadlovska, A.; Katalinic, B.; Hrubina, K. \& Wessely, E.: The Theory of Processes ...

6.3 Approximation of a partial differential equations by a system differentialalgebraic equations (DAE)

For the application of the LQ control method and the minimum principle, it is necessary to express the mathematical model of the process (9)-(11) taking the form of a system of differential equations. For this reason, we prescribe additional conditions on the function $\varphi(M, t)$ :

1) The function must satisfy the equation (9), which is approximated by a system of differential equations which is satisfied by unknown functions.

2) It must satisfy the boundary conditions (10), which are approximated by a system of linear algebraic equations.

In fact, a mixed system consisting of a system of differential and algebraic equations (DAE) supplemented by initial conditions is obtained, (Jadlovská,A. \& Jadlovská,S., 2013).

For the control desing, the reduction of the model of the evolutionary partial differential equation, (9), can be expressed in the form:

$$
\begin{aligned}
\boldsymbol{x}(k+1) & =\boldsymbol{A} \boldsymbol{x}(k)+\boldsymbol{B} \boldsymbol{u}(k)+\boldsymbol{E} \boldsymbol{z}(k), \quad \boldsymbol{x}\left(t_{0}\right)=\boldsymbol{x}_{0} \\
\boldsymbol{y}(k) & =\boldsymbol{C} \boldsymbol{x}(k)+\boldsymbol{D} \boldsymbol{u}(k)
\end{aligned}
$$

where $\boldsymbol{x}$ is the state of the model, $\boldsymbol{y}$ is its output, $\boldsymbol{u}$ is its input (a variable), $\boldsymbol{z}$ represents the ambient temperature and $\boldsymbol{A}, \boldsymbol{B}, \boldsymbol{C}, \boldsymbol{D}, \boldsymbol{E}$ are the state matrices.

\subsection{Algorithm}

The algorithm for the solution of optimum control problems for systems with distributed parameters consists of following steps:

Step 1. Input

- input values for the process model (9), (13), $\rho, c, \lambda, \sigma_{1}, \sigma_{2}, \alpha_{1}, \alpha_{2}, \varphi_{0}$,

- input values for the criterial function: $H_{\varphi}, \alpha_{s}, \varphi\left(S, t_{f}\right), \varphi\left(0, t_{f}\right), t_{f}$ (the total time of the process),

- the numerical method parameter $\varepsilon$ and the values $P_{1}, P_{2}$ (for initial control).

Step 2. Select the initial control as the function $u(t)=f\left(P_{i}, t\right)$ where $P_{i}, i=1,2$ are parameters.

Step 3. Calculate the non-linear boundary condition (an equation of order $4^{0}$ ) and the solution of the bi-dimensional partial differential equation using the finite element method.

Step 4. Calculate the criterial function value (14) using a selected numerical integration method.

Step 5. If at the time $t_{f}$ the temperature satisfies the conditions of the problem then we continue the calculation, otherwise we perform a correction of the selected control and return to step 3 .

Step 6. If the difference of the values of the criterial functions satisfies the condition for termination then terminate the computation, otherwise go on to step 7 .

Step 7. Change the values of parameters and generate a new control; then continue from step 3 . 
In step 2. the parameters $P_{1}$ and $P_{2}$ are selected during the computation process in such a way as to ensure that the value of the criterial function decreases. The control function may be formed on the basis of experimental investigation of temperature distribution inside the aggregate.

In the numerical solution of the optimum control problem for systems with distributed parameters it is our goal to determine, on the basis of the mathematical model, the temperature distribution $\varphi(S, t)$ inside the heated material and the time curve $u(t)$ of the medium temperature inside the heating aggregate to minimize the objective functional (14) when the heating process is described by the heat conduction partial differential equation (9) with non-linear boundary condition (13), the initial condition $\varphi(x, 0)=\varphi_{0}(x)$ and the condition describing the necessary temperature to be reached both on the surface and in the center of the heated material within a prescribed time $t_{f}$, i.e. $\varphi\left(S, t_{f}\right)$ and $\varphi\left(0, t_{f}\right)$. For our simulation we chose the following values:

$$
\begin{aligned}
& \lambda=29,2728\left(W \cdot m^{-1}{ }^{0} C^{-1}\right), \rho=7,488\left(k g \cdot m^{-3}\right), c=0,690822\left(\mathrm{~kJ} \cdot \mathrm{kg}^{-1}{ }^{0} \mathrm{C}^{-1}\right), a=0,56 \cdot 10^{-5}\left(\mathrm{~m}^{2} \cdot \mathrm{s}^{-1}\right), \\
& \varphi\left(S, t_{f}\right)=\left\{1250^{\circ} \mathrm{C}, 1250^{\circ} \mathrm{C}\right\}, \varphi_{0}(x)=20^{\circ} \mathrm{C}, \varphi\left(0, t_{f}\right)=\left\{1200^{\circ} \mathrm{C}, 1230^{\circ} \mathrm{C}\right\}, u(t) \leq 1400^{\circ} \mathrm{C} \\
& v(t)=0,005 \mathrm{~m} \cdot \mathrm{s}^{-1}, t \in\left\langle 0, t_{f}\right\rangle, t_{f} \in\langle 6000 \mathrm{~s}, 15000 \mathrm{~s}\rangle .
\end{aligned}
$$

If we use, for the purpose of simulation, an optimality criterion of the form (14) we select numerical values for $H_{\varphi}$ and $\alpha_{s} ;$ e.g. $H_{\varphi}=850^{\circ} \mathrm{C}$ and $\alpha_{s}=1 \times 10^{-11}$. Slab thickness $2 s=0,1 m$; heating time $t_{f}=12.250 s$.

The result of numerical solution is the determination of the time dependence of material surface and medium temperature. The simulation results for the defined input values of the heated material can be presented to the user graphically, however, for the control needs, they can be stored in a computer memory in the form of polynomials. The coefficients of approximation polynomials may be calculated by the use of the program, e.g. the least squares method. Using the mentioned procedure, for the problem to be solved we can express

Medium temperature in the furnace:

$$
u(t)=-0.0079 t^{5}+0.1838 t^{4}-1.8428 t^{3}+16.3801 t^{2}-9.9226 t+504.8695 ; \quad I=0.997
$$

Slabe surface temperature:

$$
\varphi\left(S, t_{f}\right)=-0.0227 t^{5}+0.5074 t^{4}-3.0196 t^{3}+8.5112 t^{2}+26.9025 t+21.7846 ; \quad I=1.00
$$

\subsection{On the methods and algorithm}

For the numerical solution of the defined tasks, the authors have designed and verified algorithms of the methods. Some of them are as follows: 


\section{Numerical methods}

The algorithm of the method of successive approximations using the maximum principle, the algorithm of a generalized reduced gradient method GRGM. The GRGM algorithm may be used to solve discrete tasks of the systems optimal control. Numerical experiments have showed the satisfactory convergence of the algorithm, (Hrubina, 1993), (Jadlovská et al., 2011).

2.Iterative methods of linear algebra (Jacobi algorithm, Gauss-Seidl method and relaxation method).

In (Hrubina, 1993; Hrubina \& Jadlovská, 2002) two tasks of the optimal control of the system with distributed parameters were formulated, in which the mathematical model is expressed by the operators equations and inequalities (it regards the systems control described by partial differential equations of an elliptic and parabolic type). If a simple algebraic transformation is applied to the defined tasks, we obtain classical tasks. To solve classical tasks, iterative algorithms of algebraic methods may be used, which have the advantage of a strong converge to a fixed point (a set of equations of a state control has the matrix, which is the Mmatrix).

3.Methods of hierarchical decomposition

Algorithm of admissible and inadmissible solutions method and a mixed method.

The solution of optimal control problems of systems with distributed parameters, after the mathematical model discretization, i.e. a partial differential equation with the initial and boundary conditions, leads to the optimization problem of large dimensions, which generally cannot be solved directly. After the application of the decomposition method and the hierarchy to the problem being solved, we will obtain the task which can be solved by the algorithm of the method of models coordination (Jadlovská et al., 2011), (Hrubina \& Taufer, 2005).

4.The moments method

Algorithm of the 1 - problem moments. In (Hrubina, 2003) the two tasks of optimal control of the systems with distributed parameters are formulated. There is presented a general procedure of the use of the 1-problem moments method for the solution of a variational task of optimal control in the systems which are described with partial differential equations. There is also provided an algorithm for the case of a second-order task, the numerical solution of the temporal optimization task, in which the variables are dimensionless.

5. The method of finite elements and its algorithm.

In (Hrubina \& Jadlovska, 2004) it is shown that the mathematical model of the process described by a bidimensional partial differential equation can be expressed in the form of a functional equation whose solution (if any) implements a minimum of a functional. For the rate of convergence of the equation solution, a relation was derived. For the functionals minimization, the variational principle in connection with the method of finite elements was applied, thus providing an effective tool to solve the defined tasks. The algorithm of the finite elements method can be applied to solve the tasks of a stationary and non-stationary heat conduction as well as the tasks of the processes optimal control. 
6. Algorithm based on the mini-max theorem.

A contribution of the designed algorithm based on the mini-max method and a gradient method lies in the fact that it can be applied to solve the defined task of the optimal control of the system with distributed parameters, in which the perturbations operator is considered. When solving a specific task, it is assumed that the structure and parameters of the system are known either from the documentation, or they are determined by the appropriate method, e.g. by a measurement. So in fact it is possible to create a mathematical model, which is presented by the systems of differential equations (of state and of conjugated state) and a criterial (objective) function, which ultimately are operators equations, (Jadlovská et al.,2012),(Jadlovská,A. \& Jadlovská,S. 2013).

\section{Conclusion}

The chapter contributes to the theoretical and application results of the authors' research work. It presents and deals with a modern definition of informatics, information technology, cybernetics and the theory of the optimal control of distributed parameter processes whose mathematical model is expressed by operator equations. The designed algorithm is used to solve the defined problem of the optimal control of a material heating process whose mathematical model is expressed by a partial differential equation of heat conduction, a nonlinear boundary condition and a defined functional. In addition, the chapter contains a brief characteristic of the verified algorithms for solving problems of processes optimal control.

\section{Acknowledgement}

This work has been supported by the Scientific Grant Agency of Slovak Republic under project VEGA No.1/0286/11 Dynamic Hybrid Architecture of the Multiagent Network Control Systems.

\section{References}

Athans,M., Falb,P. (1966). Optimal Control (An introduction to the Theory and Its Applications).p.867, McGraw-Hill Book Company, New York

Bellman, R. Dynamic programming. (1967). New York, Princenton University Press Butkovskij, A. G.( 1975). Methods of Control of Systems with Distributed Parameters, Nauka, Moskva

Hrubina, K. (1993). Solving optimal control problems for systems with distributed parameters by means of iterative algorithms of algebraic methods. Kybernetika a informatika, , roc. 6 , c. 1 , s. $48-64$

Hrubina, K., Jadlovská,A. (2002). Optimal Control and Approximation of Variational Inequalities. Kybernetes The International Journal of Systems and Cybernetics.Vol 9/10, Emerald England, pp.1401-1408, ISSN 492X.0368

Hrubina, K., Taufer, I. (2005). Optimal Control of Processes based on the use of Informatics Methods, Informatech Košice, , 286 s., ISBN 80-88941-30-X 
Hrubina, K. (2003). Algorithm Applications to Determine optimum Trajectories of the Controlled Process, , IFAC workshop on Programmable Devices and Systems, PDS 2003, pp. 75-78, TU Ostrava

Hrubina, K., Jadlovská, A. (2004). Optimal control problems solved by the Application of Algorithms of Numerical Method, Chapter 26, DAAAM International Scientific Book 2004, In Katalinic,B.(Ed), Vienna, Austria, pp. 265-282, ISBN 3901509-38-0, ISSN 1726-9687

Jadlovská,A., Katalinic,B.,Hrubina,K., Macurová,A., \& Wessely,E. (2011).Optimal Control of Nonlinear Systems with Constraint, Chapter 22, DAAAM International Scientific Book, In Katalinic, B.(Ed), Vienna, Austria, pp. 265-282, ISBN 968-3901509-84-1, ISSN 1726-9687

Jadlovská, A., Hrubina,K. (2011). Algorithms of optimal control methods for solving game theory problems. Kybernetes, The International Journal of cybernetics, systems and management sciences. Emerald, Vol.40 Number 1/2, pp. 290-299, ISSN 0368$492 X$

Jadlovská,A., Jadlovská,S. (2013). Modern methods of modeling and control of nonlinear systems, (in slovak). Monograph, Univ. Press elfa,Košice, p.270, ISBN 97880-8086-228-2

Jadlovská,A., Katalinic,B.,Hrubina,K., \& Wessely,E. (2012).Solution to the Problem Control of a Distributed Parameter Process, Chapter 15 DAAAM InternationalScientific Book, In Katalinic, B.(Ed), Vienna, Austria, pp. 169-186, ISBN 968-3- 901509-84-1, ISSN 1726-9687

Hulko,G., Antoniová, M., Belavý, C., Belanský, J., Szuda, J., \& Végh, P. (1998). Modeling Control and Design of Distributed Parameter systems, Publ. house

STU Bratislava, ISBN 80-227-1083-0

Kolmogorov A. N. (1965). Three approaches to the quantitative definition of information. Problems Inform. Transmission 1

Lasiecka, I.,Triggiani, R. (2000). Control Theory for Parcial Differential Equations. Cambridge University Press, ISBN 0-521-43408-4

Lions, J. L. (1986). Optimal Control of Rudder Systems by Partial Differential Equations, , Dunod Gautier-Villars, Paris

Mesarovič, M.D. (1970). Theory of hierarchical multilevel System, Academic Press, New-York

Pontrjagin, L.S., Boltyansky, V.G.,Gamkrelidze, R.V., \& Mishenko, E.F.(1983).Mathematical Theory of Optimal Processes, Interscience, New-York Poznyak, A.(2008). Advanced Mathematical Tools for Automatic Control Engineers Elsevier, Amsterdam, New-York, p.774 , ISBN 978080446745

Shannon, C.E. (1948). A mathematical theory of communication. Bell System Technical Journal, vol. 27, pp. 379-423 and 623-665

Tripathi, S.M. (2008).Modern Control Systemes (An Introduction), Infinity Science Press LLC, Hingham, Massachusetts, New Delhi, pp.227, ISBN 078-1-934015-21-6 Wiener, N. (1961). Cybernetic. MIT Press 